\title{
Modelo de muestreo comprimido multiespectral para radio cognitiva
}

\author{
Compressed sensing multiespectral model for cognitive radio networks \\ Jeison Marin ${ }^{1 *} \quad$ Leonardo Betancur ${ }^{1} \quad$ Henry Arguello $^{2}$ \\ Recibido 9 de noviembre de 2016, aceptado 31 de mayo de 2017 \\ Received: November 9, $2016 \quad$ Accepted: May 31, 2017
}

\begin{abstract}
RESUMEN
La radio cognitiva es una de las técnicas más prometedoras para optimizar el uso del espectro. Sin embargo, la gran cantidad de información espectral que es necesario analizar para identificar y asignar porciones espectrales hace que se incrementen los tiempos de asignación de canal debido al previo procesamiento de los datos y, por lo tanto, no sea posible ofrecer servicio a todos los dispositivos que lo requieran. El muestreo comprimido, por su parte, es una técnica que permite la reconstrucción de señales dispersas o compresibles usando un número menor de muestras que las requeridas por el criterio de Nyquist. En este trabajo se presenta un nuevo modelo que usa muestreo comprimido multiespectral para el sensado del espectro radioeléctrico en radio cognitiva, que mejora los tiempos de sensado y asignación de canal al disminuir el tamaño de los datos necesarios para reconstruir la información espectral de potencia a distintas bandas. Este modelo se basa en arquitecturas que usan muestreo comprimido para analizar imágenes multiespectrales. Se propone la operación de un gestor espectral centralizado que mediante patrones binarios selecciona o descarta los datos de potencia de diferentes radios definidos por software ubicados en diferentes posiciones geográficas. El gestor espectral, con base en las muestras tomadas y aplicando las técnicas de muestreo comprimido multiespectral, reconstruye un cubo de datos con la información de potencia transmitida y frecuencia de operación de todos los usuarios. Los resultados muestran que es posible esta reconstrucción utilizando solo 50\% de las muestras generadas por todos los dispositivos, y que la información se puede almacenar usando solo 6,25\% del total de datos originales.
\end{abstract}

Palabras clave: Muestreo Comprimido, radio cognitiva, sensado espectral.

\begin{abstract}
Cognitive Radio is one of the most promising techniques for optimizing the use of spectrum. However, the large amount of spectral information that must be processed to identify and assign spectral components makes the channel assignment's times to be increased due to the previous processing of this data and therefore cannot provide service to the devices that require it. Meanwhile, the compressed sampling is a technique that allows the reconstruction of sparse or compressible signals using fewer samples than those required by the Nyquist criterion. This paper presents a new model that uses compressed multispectral sampling for radio-electric spectrum sensing in cognitive radio that improves sensing and channel assignment times, decreasing the number of data required for reconstructing the power spectral information in different bands. This model is based on architectures that use a compressed sample to analyze multispectral images. The operation of a centralized manager is presented to select the power data of different software defined radios (SDR) by binary patterns. These SDRs are in different geographical

1 Programa de Ingeniería de Telecomunicaciones. Universidad Pontificia Bolivariana. Campus de Laureles, Circular 1 № 70-01. Medellín, Colombia. E-mail: leonardo.betancur@upb.edu.co

2 Escuela de Ingeniería de Sistemas e Informática. Universidad Industrial de Santander. Calle 9 Carrera 27. Bucaramanga, Colombia. E-mail: henarfu@uis.edu.co

* Autor de correspondencia. E-mail: jeison.marin@upb.edu.co
\end{abstract}


positions. The centralized manager reconstructs a data cube with the transmitted power and operation's frequency of all the users based on the samples taken and applying multispectral sensing techniques. The results show that this multispectral data cube can be built with only a 50\% of the samples generated by the devices, and can be stored using only a $6.25 \%$ of the original data.

Keywords: Compressive sensing, cognitive radio, spectrum sensing, multi-dimensional arrays.

\section{INTRODUCCIÓN}

En los sistemas de radio de nueva generación, el uso eficiente del espectro es una necesidad imperativa. El fenómeno de masificación de datos de los servicios de acceso inalámbrico móvil que es característico hoy en día tiende a aumentar de manera vertiginosa, y va en contra vía con el uso del espectro concentrado en unas bandas de comunicaciones muy específicas, principalmente en las bandas de telefonía móvil celular GSM, UMTS y LTE, las bandas de $850 \mathrm{MHz}, 900 \mathrm{MHz}$, $1900 \mathrm{MHz}, 2700 \mathrm{MHz}$ y las ISM. Gran parte de estas bandas espectrales se encuentran densamente usadas y contaminadas por interferencia, al contrario de algunas bandas de UHF que son subutilizadas, lo que en muchos casos perjudica el buen desempeño y explotación del espectro electromagnético [1-2].

La radio cognitiva $(\mathrm{RC})$ ha surgido en los últimos años como una innovadora alternativa para reutilizar las porciones del espectro que en determinado tiempo no están siendo usadas por los usuarios licenciados. A los usuarios licenciados normalmente se les denomina usuarios primarios (UP), y a los usuarios no licenciados que aprovechan las porciones no usadas de espectro se les denomina usuarios secundarios (US) [3]. En particular, la RC supone que los dispositivos inalámbricos deben ser capaces de determinar cuáles porciones del espectro están libres para entrar a ocuparlos, esto es conocido como sensado espectral. Sin embargo, cuando se trata de la RC aplicada a escenarios de alto tráfico de datos y densidad de usuarios, los requerimientos de velocidad para esta operación son mayores, de lo contrario se podrían generar tiempos de latencia altos en la ocupación o abandono del espectro por parte de los usuarios cognitivos.

En [4] se muestra un ejemplo de la incidencia que tiene el aumento de la cantidad de datos procesados durante el sensado espectral en la adecuada asignación de espectro a los usuarios cognitivos en un escenario con un área fija de $\mathrm{x} m^{2} \operatorname{con} p$ UPs transmitiendo a $P$ $\mathrm{dBm}$ por medio una estación base principal, y $s$ USs transmitiendo a $S \mathrm{dBm}$ mediante una estación base cognitiva donde $S<P$. Los UP tienen cierto tiempo de servicio, por lo que constantemente están ocupando y desocupando el espectro. Los US ocupan el espectro de una manera oportunista, no tienen asignado tiempo de servicio y deben abandonar su canal y proceder a buscar otro espacio disponible cuando un usuario primario llega. Cuando algún usuario cognitivo necesita acceder al espectro debe hacer una petición por medio de un canal de control común a un servidor o gestor espectral, enviándole la información espectral que muestrea en un paquete de control, para luego esperar que le sea asignada una porción espectral libre de interferencia. Si dos o más usuarios cognitivos hacen una petición cuando el canal de control está ocupado, sucede una colisión. La Figura 1 muestra el desempeño del sistema, en términos de número de colisiones vs. duración de transmisión de paquetes de control para una instancia del problema.

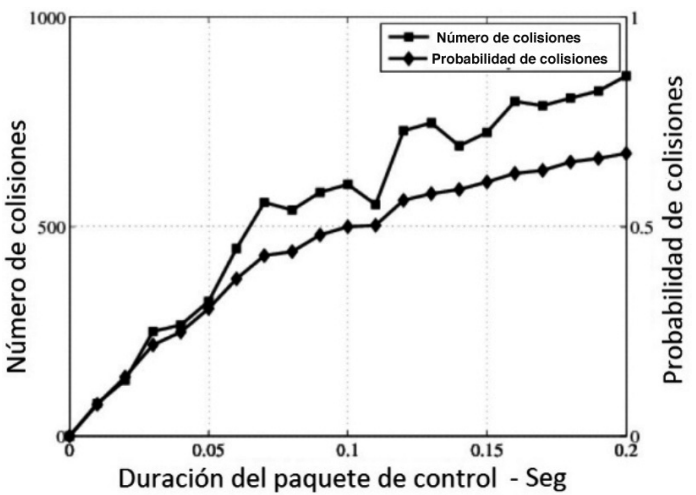

Figura 1. Número y probabilidad de colisiones con respecto a la duración de los paquetes de control. Tomado de [4].

Se puede observar que a medida que aumenta la duración de transmisión de los paquetes, aumentan las colisiones. Más cantidad de datos por procesar 
produce más colisiones y, por lo tanto, un peor desempeño, ya que el US permanece sin servicio o interfiriendo con un UP mientras logra acceder al canal de control. Adquirir una señal usando pocas muestras y luego recuperarla con un error mínimo, hará que toda la información espectral y de control que se manipula en RC ocupe menos memoria, mejorando así la velocidad de procesamiento y transferencia.

Una tecnología que desde el 2004 ha llamado la atención de investigadores en el campo del procesamiento de las señales es el muestreo comprimido (MC), [5-10]. La implementación y desarrollo de $\mathrm{MC}$ ha tenido un gran crecimiento en los últimos 10 años [5]. Esta técnica permite, bajo ciertas condiciones, usar un número muy reducido de muestras durante el sensado de una señal, y luego reconstruir la señal con el mínimo error. El MC ha tenido aplicaciones en diversas áreas como procesamiento de imágenes, imágenes médicas, datos sísmicos, aplicaciones biológicas o radares [11]. El tema ha despertado igualmente el interés en investigaciones del área de las telecomunicaciones [12-13], como en estimación de canal inalámbrico [14], canales MIMO [15], canales OFDM [16], sistemas UWB [17], redes de sensores [18], antenas [19], y redes P2P [20], entre otras.

Asimismo, el MC ha sido ampliamente usado en RC. Por ejemplo, en [21], se discute un modelo general MC aplicado a RC donde se submuestrea una señal espectral aprovechando su dispersión en una base de representación wavelet. Bajo este mismo esquema, en [22], se comparan diversas metodologías de MC para mejorar el desempeño en la reconstrucción de la señal como BP-PD (Basis pursuit with primal dual interior point method), BP-SPG (Basis pursuit with spectral projected gradient) ROMP (The regularized Orthogonal Matching Pursuit) y reconstrucción adaptativa. Finalmente, en [23], se presenta un modelo cooperativo de red virtual, con ubicación espacial de UP's y US's y un nivel de potencia asociado a cada uno.

Una rama de aplicación de MC es el muestreo y reconstrucción de imágenes multiespectrales. Técnicas nombradas como Compressive Spectral Imaging (CSI), han sido propuestas en una variedad de arquitecturas durante los últimos años [24-27]. Con estas técnicas es posible obtener imágenes espectrales 3D de alta resolución y ser captadas sobre un plano focal 2D mediante una cámara digital, eliminando un alto porcentaje de muestras, y luego usa el arreglo 2D para recuperar nuevamente el cubo de la imagen.

En este trabajo se aplican los avances conseguidos en estas técnicas de MC y CSI, en una red inalámbrica cognitiva. De forma similar a las técnicas CSI, se presenta un modelo multiespectral con planos de información de intensidad de potencia georreferenciada a diferentes frecuencias, con el objetivo de lograr alta compresión de los datos.

Esta forma de modelar la ocupación espectral se conoce en algunos contextos como mapas de radiofrecuencia, mapas de densidad espectral de potencia o cartografía de interferencia [28-30]. En [28-29] se usan mapas con información sobre niveles de interferencia en ciertas áreas geográficas con el objetivo de usar algoritmos de interpolación para predecir la potencia en toda el área, usando información parcial obtenida por algunos dispositivos. Sin embargo, en estos trabajos no se aborda la compresión durante el muestreo de los datos, y cada mapa se analiza y reconstruye en forma independiente. En [30-31] se introducen las técnicas de muestreo comprimido para la construcción de los mapas de cartografía de interferencia, y en [32] se resumen las técnicas usadas para la elaboración de estos mapas. A diferencia de estos trabajos, en este artículo se propone un modelo que no solo explota la dispersión espacial, sino además la espectral, dimensionando estos mapas como cubos multiespectrales, lo que permite adaptar técnicas CSI y aprovechar sus bondades en la compresión y reconstrucción de datos. Esto permite incrementar la razón de diezmado de información cuando se trabaja con varios planos. En el modelo propuesto se reduce la cantidad de datos en dos etapas: en el momento del muestreo de la señal por parte de los dispositivos, y en el momento del procesamiento de las muestras tomadas. En un arreglo 2D queda condensada toda la información necesaria, para luego construir el cubo de datos con información de potencia en el dominio del espacio, a diferentes bandas espectrales.

El resto del artículo se organiza como sigue. En la sección 2 se presentará la teoría de MC y su aplicación en imágenes multiespectrales, con el propósito de mostrar su similitud con el problema de identificación espectral en RC, lo que será discutido en la sección 
3. En la sección 4 se presentará el modelo de MC multiespectral para RC. Un aspecto importante de este nuevo modelo es la acción de un gestor espectral, el que implementará el MC con base en unos patrones binarios que definirán que muestras descartar o no durante sensado espectral, por lo que en la sección 5 se discutirán las arquitecturas propuestas para la implementación del MC usando dichos patrones. Finalmente en la sección 6 se analizan los resultados de la implementación del modelo en un ambiente de simulación junto con los criterios de calidad en la reconstrucción del espectro.

\section{MUESTREO COMPRIMIDO Y SU APLICACIÓN EN IMÁGENES MULTIESPECTRALES}

\section{Muestreo comprimido}

En el MC la compresión de una señal se realiza durante su muestreo, el que puede realizarse por debajo del límite establecido por el teorema de Shannon-Nyquist [33], debido a principios teóricos como la dispersión e incoherencia de la señal o de una transformada de la señal [5]. En cuanto a la propiedad de dispersión, sea una señal discretizada, representada en forma discreta por un vector $s \epsilon$ $\mathbb{R}^{N}$. Se dice que $s$ tiene un factor de dispersión $K$ si $\|s\|_{0}=\leq K$, donde $\|s\|_{0}=\#\{i: x(i) \neq 0\}$, y $s$ contiene elementos no nulos de la señal. Las señales con baja dispersión, es decir, con un factor $K$ muy alto, comúnmente se convierten a señales con mayor dispersión mediante transformaciones lineales de la forma $f=\Psi_{s}$ donde $\Psi \in \mathbb{R}^{M \times N}$ corresponde a una base de representación, como por ejemplo una base de transformada Wavelet, transformada Coseno, transformada de Fourier o una base Kronecker [34, 35]. Los también denominados diccionarios $\Psi$ de forma alternativa pueden construirse mediante procesos de aprendizaje o entrenamiento desde los datos originales cuando las bases existentes no satisfacen condiciones de dispersión necesitadas [36-38].

Sea una señal $K$-dispersa $f \in \mathbb{R}^{N}$ que se muestrea de forma compresiva para obtener la señal $g \in \mathbb{R}^{M}$ donde $M<<N$. Este muestreo se puede representar de forma matricial como

$$
g=\Phi f
$$

donde $\Phi \in \mathbb{R}^{M \times N}$ se denomina matriz de muestreo del sistema.
Ahora, note que en el caso de que se desconozca $f$, dado que $M<<N$ se conforma un sistema de ecuaciones lineales indeterminado, el cual por lo general tiene infinitas soluciones. En el MC, se busca obtener una solución única para este sistema resolviendo el problema de optimización dado por

$$
\min _{f}\|f\|_{0} \text { sujeto a } g=\Phi f,
$$

Donde $f \in \mathbb{R}^{N}$ es la señal $K$-dispersa que debe ser calculada, $\Phi \in \mathbb{R}^{M \times N}$ es la matriz de muestreo, y $g \in \mathbb{R}^{M}$ corresponde a las muestras conocidas.

Sin embargo, matemáticamente este problema de optimización puede resultar de muy alta complejidad por su naturaleza no convexa, razón por la que se han desarrollado otras alternativas de solución, algunas con base en algoritmos que resuelven problemas de optimización convexa. El algoritmo de solución en el caso de programación lineal (LP) [39], resuelve

$$
\min _{f}\|f\|_{1} \text { sujeto a } g=\Phi f
$$

Por su parte, algoritmos como el Algoritmo de Nesterov (NESTA) [40], o el Algoritmo de proyección espectral de gradiente para $l_{1}$ (SPGL1) [41], resuelven el problema

$$
C_{n}=\frac{a \Delta t}{\Delta x}=\frac{a N \Delta t}{L} \leq 1.0
$$

Se pueden también mencionar algoritmos como el Algoritmo de iteraciones rápidas para disminución del umbral (FISTA) [42], el Algoritmo de proyección de gradiente para reconstrucción de señales sparse (GPSR) [43], y el Algoritmo de dos pasos iterativos de disminución de umbral (TwIST) [44], los que resuelven el problema

$$
\min _{f}\left(\frac{1}{2}\|\Phi f-g\|_{2}^{2}+\lambda\|f\|_{1}\right)
$$

Finalmente se tiene el algoritmo constrained split augmented Lagrangian shrinkage algorithm (C-SALSA) [45], el que ha sido ampliamente usado durante los últimos años debido a sus mejores características de velocidad y calidad en la solución del problema planteado en algunas aplicaciones en 
comparación con métodos ya mencionados como FISTA o TwIST. En C-SALSA el problema de optimización restringido se formula de la siguiente manera

$$
\begin{gathered}
\min _{u, w \in \mathbb{R}^{N}, v \in \mathbb{R}^{M}}\|w\|_{1}+\iota_{E(\in, 0)}(v) \\
\text { sujeto a } w=f y v=\Phi f-g
\end{gathered}
$$

donde

$$
E(\in, p)=\left\{g \in \mathbb{R}^{M}:\|f-p\|_{2} \leq \in\right\}
$$

es una elipsoide que corresponde al conjunto de soluciones factibles de la ecuación (4), y E $(\in, 0)$, por lo tanto, es una esfera euclidiana de radio $\in$ centrada en el origen de $\mathbb{R}^{D}, y \iota_{s}: \mathbb{R}^{D} \rightarrow \overline{\mathbb{R}}$ denota la función indicadora del conjunto $S \subset \mathbb{R}^{D}$,

$$
\iota_{s}(s)=\left\{\begin{array}{c}
0 \text { si } \in S \\
+\infty \text { si } s \notin S
\end{array}\right.
$$

\section{Imágenes espectrales comprimidas}

Una imagen de un objeto se puede representar de forma espectral cuando los datos de la imagen corresponden a la radiancia y reflectancia de la superficie del objeto. Estos datos pueden ser vistos como una dispersión de puntos en un espacio Euclidiano $L$-dimensional, donde $L$ es el número de bandas espectrales. Cada pixel de una imagen espectral $v_{1}$ se puede representar como un vector $\mathrm{W}=\left[v_{1}, v_{2}, \ldots v_{L}\right]$ lo que corresponde a una firma espectral, cuya información depende del comportamiento espectral en cuanto a radiancia y reflactancia de los materiales que conforman los diversos objetos en la escena a diferentes longitudes de onda [46, 47]. Por ejemplo, una imagen RGB se puede descomponer en tres bandas espectrales que corresponden a las regiones visibles del espectro R, G y B. Sin embargo, es posible descomponer una imagen en más bandas, existiendo imágenes con decenas de bandas denominadas imágenes multiespectrales e imágenes con cientos de bandas denominadas imágenes hiperespectrales [48]. Entre más bandas espectrales tenga una imagen, mayor es su tamaño en pixeles, razón por la que el volumen de datos es enorme cuando se trabaja con este tipo de imágenes. Debido a la necesidad de disminuir el número de datos necesarios para representar una imagen espectral, en la última década se han desarrollado métodos de adquisición o captura de este tipo de imágenes utilizando la teoría de MC, aprovechando la alta correlación entre bandas y, por lo tanto, la alta redundancia de datos, y la posibilidad de representar las imágenes en bases con alta dispersión como bases Wavelet o coseno 2D.

Existen diversas arquitecturas que se han desarrollado para hacer MC a una imagen espectral en el momento de su adquisición por parte de sensores espectrales [21-24]. En estas técnicas, nombradas en la literatura como Compressive Spectral Imaging (CSI), se representa en forma discreta la información espacial y espectral de una escena a través de un cubo de datos $3 D \mathrm{f} \in \mathbb{R}^{M \times N}$ donde $M \times N$ corresponde a la dimensión espacial y $L$ es el número de bandas espectrales. Los equipos CSI capturan la imagen usando filtros binarios de apertura que permiten o se oponen al paso de la luz, realizando así el MC. Posteriormente, la información se proyecta en un plano focal que matricialmente se representa como un arreglo 2D denominado Focal Plane Array (FPA). Usando las técnicas mencionadas de MC, el cubo de datos original se reconstruye a partir del FPA.

Una de las primeras arquitecturas reportadas es la codificación compresiva en el espacio espectral para imágenes hiperespectrales (SSCSI) [23]. En esta arquitectura se reconstruye una imagen hiperespectral de alta resolución a partir de unas muestras tomadas por un único sensor ubicado en una cámara óptica que muestrea la imagen en el espacio y espectro. Un elemento que dispersa la luz es localizado después de un lente. La rejilla de difracción es seguida por otro lente y luego una máscara o matriz de muestreo que modula la imagen antes de proyectarla en un "arreglo planar" 2D. Por lo tanto el FPA, que en este trabajo es nombrado como una proyección codificada HS, se modela en forma discreta como

$$
Y=\sum_{k=1}^{p} \Phi_{k} h_{k}
$$

donde $Y$ corresponde al FPA en forma vectorizada, $h$ representa la imagen espectral original en forma vectorizada y $\Phi$ es una matriz de modulación que representa la máscara de apertura.

Posteriormente en la técnica CASSI [21] se convierte de un cubo de datos tridimensional en un plano 
focal bidimensional, es decir, un cubo de tamaño $N \times N \times L$ queda representado por un plano de $V \times N$ pixeles, donde $V=N+L-1$. En CASSI las proyecciones medidas pueden ser modeladas como $y=H f$, donde $H$ es una matriz cuya dimensión es $\mathrm{N}(\mathrm{N}+\mathrm{L}-1) \times(N \cdot N \cdot L)$, que corresponde al FPA. Matemáticamente tiene esa estructura, dado que se está modelando el efecto físico y dispersivo de un prisma en el sistema. En forma discreta, el FPA se modela como

$$
Y_{j l}=\sum_{k=0}^{L-1} F_{j(l+k)(k)} T_{j(l+k)}+w_{j l},
$$

donde $F$ es el cubo de datos espectral con $L$ bandas espectrales y $N \times N$ pixeles, $Y_{j l}$ es la intensidad medida en la posición $j, l$ del detector cuyas dimensiones son $N \times(N+L-1), T_{j l}$ es la apertura binaria y $w_{j l}$ es el ruido del sistema.

En CASSI el modelo matemático de las proyecciones se formula como $y=A \theta$ donde $A=H \Psi$ corresponde a la matriz de muestreo. Note que de acuerdo con esta representación se tiene que $f=\Psi \theta$, lo que indica que $\theta$ es una representación de $f$ en la base $\Psi$. Finalmente, en [21] muestra que el cubo de datos original es reconstruido resolviendo el problema de optimización $\min _{\theta}\left(\|y-H \Psi \theta\|_{2}+\lambda\|\theta\|_{1}\right)$ el que es análogo al problema presentado en la ecuación (5).

En las Figuras 2 y 3 se pueden observar de forma general los componentes físicos de las arquitecturas SSCSI y CASSI, respectivamente.

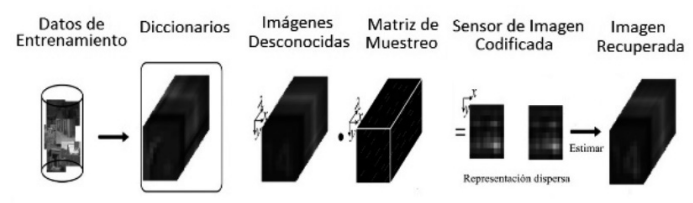

Figura 2. Arquitectura SSCSI.

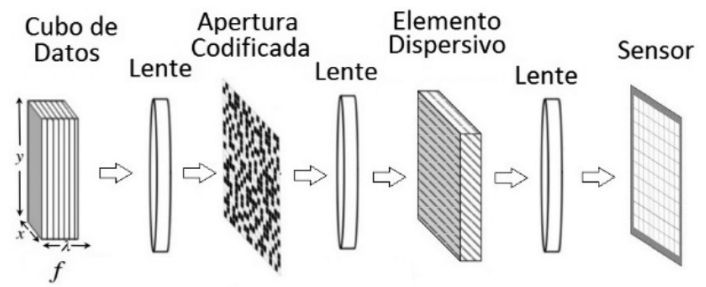

Figura 3. Arquitectura CASSI.

\section{ANALOGÍA ENTRE ARQUITECTURAS CSI Y EL SENSADO ESPECTRAL PARA RC}

En este trabajo se propone aplicar las técnicas de MC y CSI en una red inalámbrica cognitiva. Considere un escenario donde se tienen $l$ usuarios o dispositivos inalámbricos ubicados en un área geográfica de dimensión $\mathrm{M} \times \mathrm{N}$ metros cuadrados, transmitiendo información en $L$ bandas espectrales. Cada usuario tiene asignado una ranura de frecuencia o banda espectral. Para evitar interferencia, usuarios cercanos entre sí deben tener bandas diferentes. Pueden existir UP y también US que aprovechan el uso del espectro de manera oportunista cuando los UP no usan su espectro asignado. De acuerdo con esto, es posible modelar la ocupación espectral junto con la posición geográfica de usuarios o dispositivos inalámbricos en un área de cobertura, como un problema multiespectral, y con base en las técnicas conocidas de MC y CSI, proponer una nueva técnica de MC Multi-Espectral para RC que denominaremos: "Compressive Spectral Cognitive Radio" (CSCR), con el fin de realizar el sensado espectral y el reconocimiento de las bandas vacantes u ocupadas, usando menor cantidad de datos.

De forma general, a continuación se establece un paralelo entre algunos componentes de las arquitecturas CSI y CSCR:

- En CSI la imagen se modela como un cubo de datos donde cada pixel le corresponde una posición espacial $(x, y)$ y una firma espectral que se puede representar con el vector $\Phi=\left[\lambda_{1}\right.$, $\left.\lambda_{2}, \ldots, \lambda_{L}\right]$. En CSCR cada usuario cognitivo se encuentra ubicado en un punto $(x, y)$ y es capaz de sensar el espectro en un ancho de banda dividido en $L$ slots de frecuencia, cuyas frecuencias centrales se pueden representar por un vector $\lambda_{x y}=\left[\lambda_{x y 1}, \lambda_{x y 2}, \ldots, \lambda_{x y \mathrm{~L}}\right]$.

- En CSI cada pixel tiene una intensidad cuyo color depende de la banda espectral en la que se encuentre. En CSCR cada usuario cognitivo está transmitiendo a un nivel de potencia en $\mathrm{dBm}$, cuya intensidad depende de la banda que le fue asignada para transmitir. Por lo tanto, lo que en CSI son intensidades cromáticas en CSCR son intensidades de potencia.

- En CSI se trabaja alrededor del espectro visible electromagnético, mientras que en CSCR se 
trabaja en el rango de las ondas de radio hasta las microondas.

- En CSI se usan aperturas codificadas para realizar el MC. Estas aperturas permiten o no el paso de la luz en las cámaras digitales multiespectrales. En CSCR de forma análoga se utilizarán patrones binarios que definirán que intensidades de potencia muestrear y en que bandas espectrales.

- En CSI existe un sensor que recoge las proyecciones de la imagen en una FPA, y se representa luego matricialmente como un arreglo de dimensión 2D. En CSCR existe un gestor espectral que construirá un arreglo planar de potencia (PSPA: Power Signal Plane Array) con las muestras de potencia en función de la banda espectral captada.

- En CSI se cuenta con las muestras compresivas proyectadas en el FPA y con base en estas muestras se recupera la imagen original resolviendo el problema de optimización anteriormente descrito. En CSCR se cuenta con un gestor espectral que construye el PSPA y con base en él recupera el cubo original con la información geográfica y espectral de todos los usuarios cognitivos resolviendo el mismo problema de optimización. En ambos casos tanto la imagen espectral como el cubo espectral original son desconocidos.

\section{MODELO DE MUESTREO COMPRIMIDO MULTIESPECTRAL PARA RADIO COGNITIVA - CSCR}

\section{Escenario propuesto}

El escenario propuesto consiste en un grupo de dispositivos inalámbricos tratando de acceder a recursos espectrales, donde algunos son UP o usuarios licenciados, y otros son US o usuarios cognitivos, como se muestra en la Figura 4.

Los usuarios cognitivos solicitan recursos espectrales a un gestor espectral. El gestor espectral define cuáles bandas espectrales están vacantes u ocupadas de acuerdo con la información espectral parcial que le envía cada usuario cognitivo.

\section{El gestor de espectro}

El sistema central que recibe la información espectral de los Radios Definidos por Software (SDR: Software Define Radio) y la posterior asignación espectral será

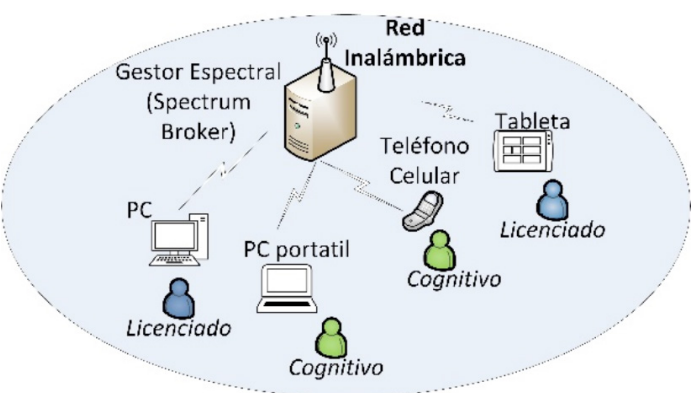

Figura 4. Escenario base.

un gestor de espectro centralizado. $\mathrm{Al}$ respecto, en la literatura se ha propuesto la intervención de una entidad generalmente conocida como Spectrum Broker (SB) o Bandwidth Broker (BB), la que controla y distribuye los recursos espectrales [3,51]. El SB debe implementar decisiones y algoritmos de control usando un paquete de software llamado Motor Cognitivo [52]. Los gestores espectrales, que son aún un área abierta de discusión [53], son formalmente usados en aplicaciones cognitivas reales. Por ejemplo, en [54], un BB es usado para asignar ancho de banda a unos usuarios dentro de una femtocelda. De acuerdo con el contexto en que un SB es usado y las funciones que implementa, toma nombres como Bandwidth Broker [55], Spectrum Policy Server [51], Regional Spectrum Broker [53] o simplemente Broker [56]. En todos estos escenarios, el SB es la entidad responsable de ejecutar tres de las cuatro funciones básicas definidas para radio cognitiva: Seleccionar los canales disponibles (Spectrum Decision), coordinar el acceso a estos canales entre usuarios disponibles (Spectrum Sharing), y desocupar los canales cuando un UP es detectado (Spectrum Mobility) [3].

\section{Modelo general de ocupación multiespectral}

Este modelo se basa en concebir la información de potencia transmitida de los diferentes usuarios licenciados y cognitivos en las diferentes frecuencias asignadas, como un cubo de datos multiespectral. Para desarrollar el planteamiento, la Figura 5 muestra $s$ dispositivos inalámbricos cognitivos que requieren servicio ubicados en un área geográfica. Note que un usuario nuevo que no se le ha asignado banda de frecuencia para transmitir entra a solicitar el servicio. Este usuario le envía un requerimiento al SB, el cual decide cuáles bandas de frecuencia están libres luego de analizar la información espectral que recibe de los demás usuarios cognitivos del sistema. 
Suponga que en cierto instante todos los usuarios están transmitiendo a determinada potencia, en alguna banda de frecuencia previamente asignada. Hay $L$ posibles slots de frecuencia a ser usados. Se define una imagen $f_{k}$, donde $1 \leq k \leq L$, como la imagen de intensidades de potencia en la frecuencia $k$. La Figura 6 muestra un ejemplo con 20 usuarios, donde algunos están transmitiendo en la banda espectral $1(B 1)$, otros en $B 2, B 3$ y $B 4$. Note que los usuarios transmitiendo en una banda conforman una imagen espectral propia. El nivel de gris de las cuatro bandas mostradas en la Figura 6 denota la intensidad de potencia.

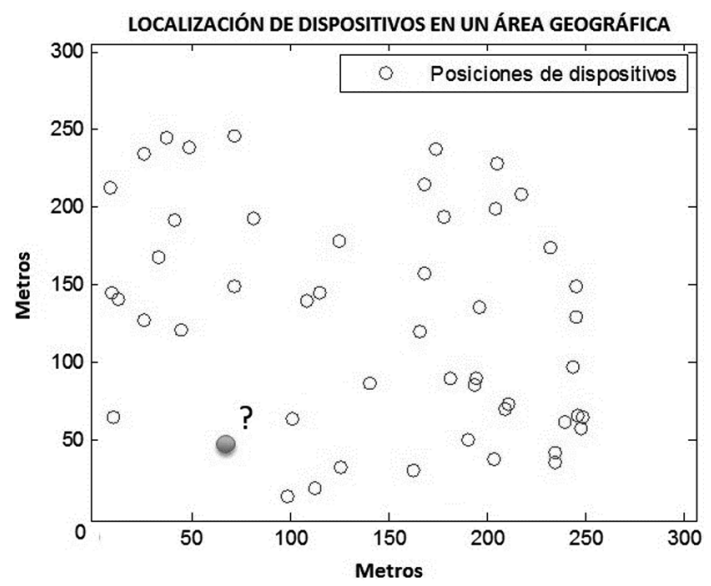

Figura 5. Área geográfica con dispositivos inalámbricos.
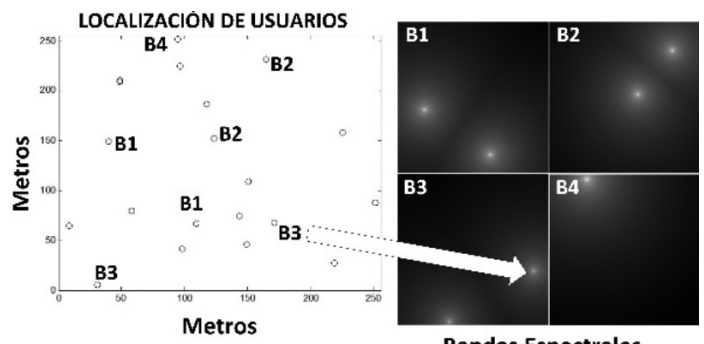

Usuarios

Bandas Espectrales

Figura 6. Imágenes de intensidades de potencia para cuatro bandas de frecuencia.

Para $L$ ranuras de frecuencia disponibles para asignar, es posible definir $L$ imágenes. Considere un ejemplo donde $L=8$ y se le debe dar servicio a los usuarios mostrados en la Figura 6, por lo tanto, estos se deben distribuir en las 8 ranuras de frecuencia. Como algunos usuarios van a quedar en la misma banda se debe garantizar que están adecuadamente distanciados entre sí para no generar interferencia. Las $L$ imágenes se pueden ver como un cubo multiespectral, como se muestra en la Figura 7.

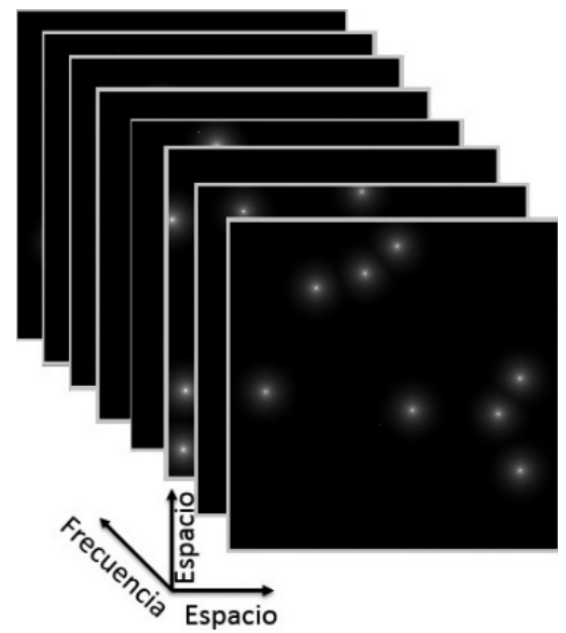

Figura 7. Cubo de datos multiespectral de intensidades de potencia.

Formalmente, sea un cubo de datos $f(x, y, \lambda)$ donde $(x, y)$ corresponde a la posición geográfica de un SDR en un área determinada y $\lambda$ corresponde a un valor de frecuencia. El voxel $f\left(x, y, \lambda_{k}\right)$ corresponde a la intensidad de potencia registrada en la posición $(x, y)$ a una frecuencia $\lambda_{k}$. El nivel de intensidad de potencia se modela como un nivel de escala de grises, desde blanco (nivel máximo) hasta negro (nivel mínimo).

\section{Construcción del cubo multiespectral por parte del SB}

En la práctica, para conformar un cubo como el mostrado en la Figura 7, considere un SDR ubicado en la posición (x,y), donde éste tiene la capacidad de muestrear la señal espectral. El usuario tiene información de potencia en cada frecuencia para su propia posición, la que depende de sí mismo y de usuarios en otras posiciones. Entre más lejano esté otro usuario de él, menor es la potencia que registra de ese usuario. Note que cada SDR ofrece información de potencia en cada banda de frecuencia $\lambda_{k}$ para $1 \leq k \leq L$ en una posición $(x, y)$, como se muestra en la Figura 8. De acuerdo con la teoría de imágenes multiespectrales, la información espectral de cada usuario cognitivo correspondería a una firma espectral. 
Si hay $k$ dispositivos inalámbricos cognitivos, cada uno enviará información espectral correspondiente a su posición. Debido a que se tiene el sistema modelado como un cubo multiespectral se pueden adaptar las técnicas SCI mencionadas. El algoritmo 1 muestra las funciones necesarias para la implementación del modelo CSCR por parte de un SB, el cual con base en las muestras compresivas tomadas, reconstruye el cubo multiespectral con información de potencia y frecuencia de operación de cada SDR.

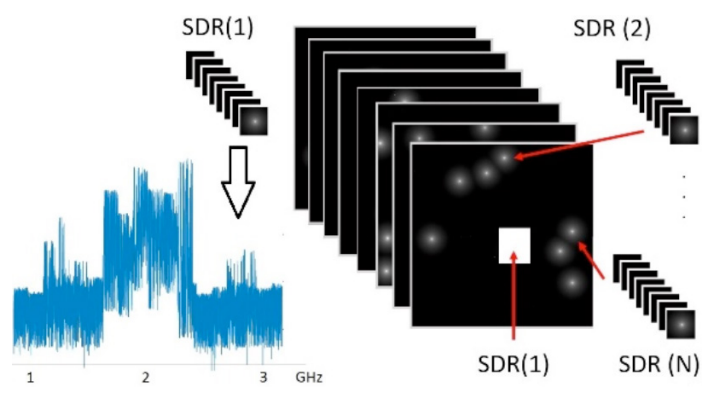

Figura 8. Conformación del cubo de datos con base en la información espectral de cada SDR.

Note en el algoritmo 1 que en los pasos 2 y 5 el SB define cuáles muestras solicitar a cada SDR para implementar el MC. Esto se hará por medio de patrones binarios, como se explicará en la siguiente sección.

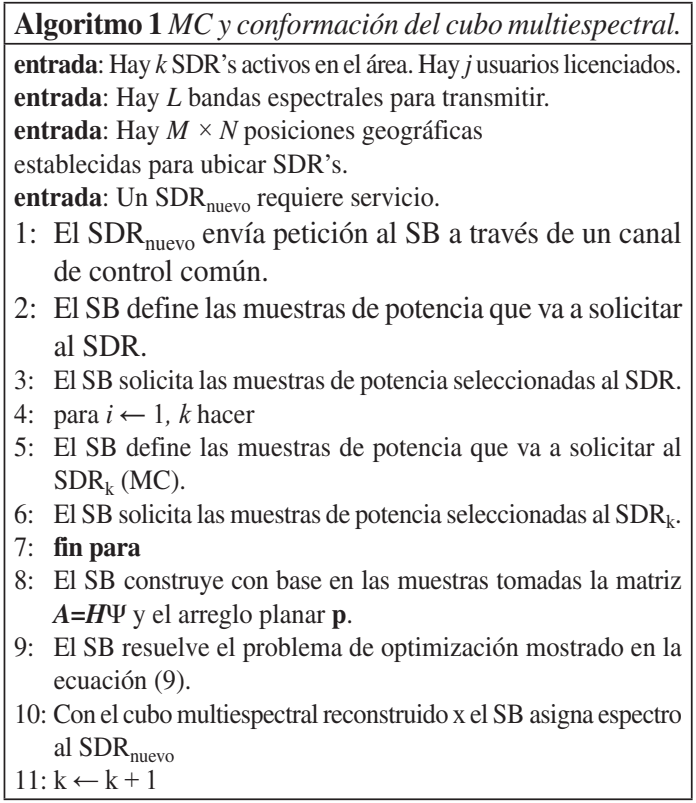

\section{ARQUITECTURAS BASADAS EN PATRONES BINARIOS}

\section{Patrones binarios y transmitancia}

El objetivo es que el SB coordine el muestreo espectral de los SDR, solicitando solo un conjunto de muestras espectrales de cada uno. En el MC, a diferencia de los esquemas de compresión de datos tradicionales, la compresión se hace durante la medición. Para definir las muestras que se deben tomar o descartar, se propone un modelo basado en patrones binarios en analogía con las aperturas codificadas de los sistemas CSI. Los patrones binarios son arreglos vectoriales o matriciales binarios que definen qué muestras tomar y qué muestras descartar de un cubo multiespectral como el mostrado en la Figura 7. El diseño de los patrones binarios lo hace el SB y se modela matricialmente como un arreglo $\mathrm{D}=\left(d_{i, j}\right) \in \mathrm{C}^{\mathrm{n}_{1} \times \mathrm{n}_{1}}, d_{i, j} \in\{0,1\}$, donde $d_{i, j}$ $=1$ representa un elemento que habilita el muestreo, llamado elemento transmisivo y $d_{i, j}=0$ representa un elemento que bloquea el sensado, llamado elemento de bloqueo. El porcentaje de elementos transmisivos en un patrón binario se puede medir calculando la transmitancia del patrón binario, definida como

$$
t_{r}=\sum_{i=0}^{n_{1}-1} \sum_{i=0}^{n_{2}-1} \frac{d_{i, j}}{n_{1} n_{2}},
$$

Donde $n_{1}$ representa la cantidad de pixeles horizontales y $n_{2}$ representa la cantidad de pixeles verticales del patrón binario. Entre mayor sea el valor de $t_{\mathrm{r}}$ más muestras son tomadas por el SB. La Figura 9 muestra tres patrones binarios a diferentes transmitancias.

La Figura 10 muestra un ejemplo de un patrón binario de 16 pixeles que define las muestras a adquirir de un arreglo que representa la información de potencia

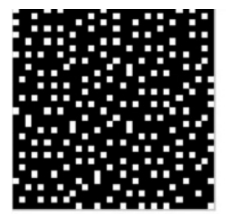

(a)

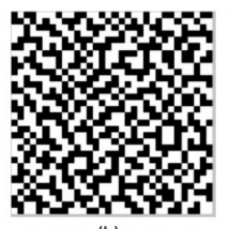

(b)

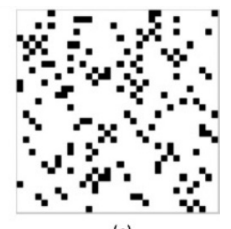

(c)
Figura 9. Patrones binarios con diferentes valores de transmitancia (a) $t_{r}=0,2$ (b) $t_{r}=0,5$ (c) $t_{r}=0,9$. 
que registran un grupo de usuarios cognitivos en la frecuencia $\lambda$. Note que de 16 posibles muestras de potencia, el código descartó 9 .

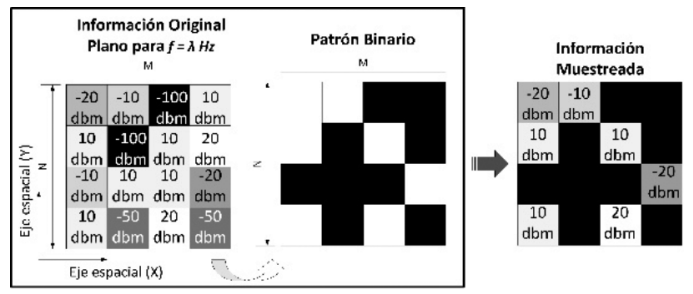

Figura 10. Efecto del patrón binario en el MC.

A continuación se presentarán tres posibles arquitecturas para implementar el uso de patrones binarios. De forma general, estas se componen de tres etapas.

1. Muestreo comprimido: El SB genera uno o varios patrones binarios que definirán el muestreo comprimido a realizar. Por medio de un canal de control el SB solicita las muestras a los SDR's. Los SDR's envían las muestras de potencia a las frecuencias seleccionadas de acuerdo con la estructura de los patrones binarios.

2. Conformación de un arreglo planar de potencia (PSPA): Con base en las muestras tomadas, el SB construye una matriz 2D realizando combinaciones lineales de las intensidades de potencia muestreadas.

3. Reconstrucción del cubo de datos: Usando técnicas de optimización aplicadas a MC y CSI se reconstruye el cubo de datos y el SB identifica las bandas espectrales disponibles y su posición geográfica.

Estas arquitecturas solo cubren la etapa de muestreo e identificación espectral en RC. Posteriormente el SB debe asignar y administrar el espectro, usando técnicas reportadas en la literatura que no son el objetivo de este trabajo, como las descritas en [3].

La primera arquitectura se basa en un patrón binario fijo para todo el cubo multiespectral. En la segunda arquitectura se propone un sistema conformado por $L$ patrones binarios, uno aplicado a cada matriz $f(\mathrm{x}, \mathrm{y}, \lambda)$ para $1 \leq k \leq L$ donde $L$ es el número de bandas espectrales disponibles. En esta segunda arquitectura se descartan muestras espaciales y también espectrales. Finalmente se propone una arquitectura con patrón binario fijo y desplazamiento del cubo espectral, como analogía al proceso denominado CASSI en CSI.

\section{Arquitectura con patrón binario fijo}

La Figura 11 muestra una arquitectura con patrón binario fijo. El SB genera un patrón binario y de acuerdo con su estructura le indica a cada SDR las muestras que éste debe enviarle. Las posiciones de los pixeles del patrón binario corresponden a posiciones geográficas. Por lo tanto, si un pixel del patrón binario es cero (representado con el color negro en la Figura 11) se está eliminando por completo la información de esta posición. Esto significa que el SB le solicitará a todos los SDR's información de potencia de solo unas posiciones geográficas seleccionadas, a todas las frecuencias espectrales.

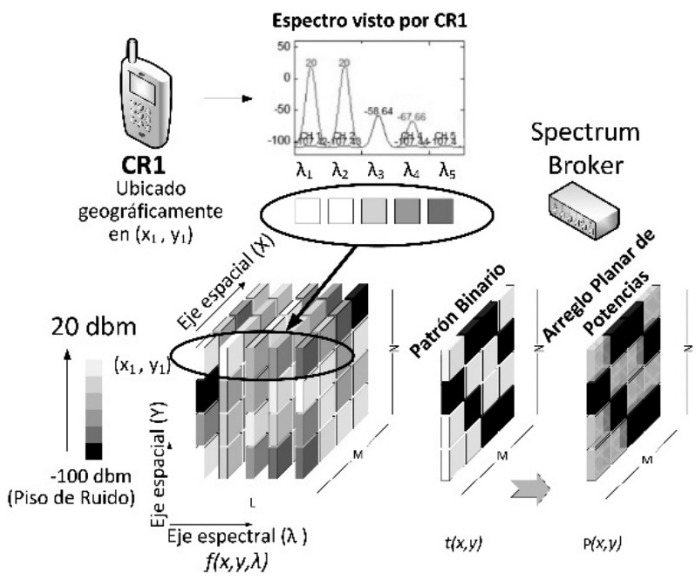

Figura 11. Arquitectura 1: Patrón binario fijo.

Note en la Figura 11 que las muestras de potencia de cada SDR son mapeadas en un arreglo planar $\mathrm{P}(x, y)$ de dimensión $M \times N$. Esto se hace mediante combinaciones lineales entre los valores de potencia enviados por los SDR y los pixeles del patrón binario. El PSPA se modela en forma discreta como

$$
P_{j l}=\sum_{k=0}^{L-1} F_{j l k} T_{j l}+w_{j l},
$$

donde $\mathrm{P}_{j l}$ es una medida de potencia proporcional a la firma espectral de la potencia registrada en la posición geográfica $(j, l)$ por los SDR, mapeada en 
el arreglo planar de potencia $\mathrm{P}$ de dimension $M \times N$; $L$ es el número de bandas espectrales disponibles para asignar a los SDRs, $T_{j l}$ es el patrón binario y $w_{j l}$ es el ruido del sistema. Note la analogía con la ecuación (9). El SB debe realizar el procesado de la señal que conforme una matriz de muestreo $H$ y posteriormente debe reconstruir el cubo de datos con la información geográfica y espectral de potencia. La reconstrucción consiste en recuperar la señal original con base en las medidas resolviendo el problema de optimización (6). Los detalles matemáticos del proceso de construcción de $H$ se pueden consultar en [21-22].

\section{Arquitectura con múltiples patrones binarios}

La Figura 12 muestra una arquitectura con múltiples patrones binarios, uno asignado a cada banda espectral con frecuencia central $\lambda_{k}$ para $0 \leq k \leq L-1$.

El SB debe generar $L$ patrones binarios diferentes. Por lo tanto, en esta arquitectura es menor la probabilidad de omitir por completo la información de un punto geográfico y se tendrá información parcial de la mayoría. En la Figura 12 se muestra un ejemplo de un SDR que aporta información de potencia en la posición $\left(x_{k}, y_{k}\right)$ donde $L=5$ y hay 5 patrones binarios. Note que de acuerdo con la estructura de los patrones binarios en el proceso de MC se omite la información de potencia de $\lambda_{2}$ y $\lambda_{4}$ para la posición $\left(x_{k}, y_{k}\right)$. De la misma forma se omitirá información espectral de otras bandas, para otros puntos geográficos $(x, y)$. El PSPA se modela en forma discreta como

$$
P_{j l}=\sum_{k=0}^{L-1} F_{j l k} T_{j l k}+w_{j l},
$$

donde de forma similar a la arquitectura de patrón binario único, $P_{j l}$ es una medida en la posición geográfica

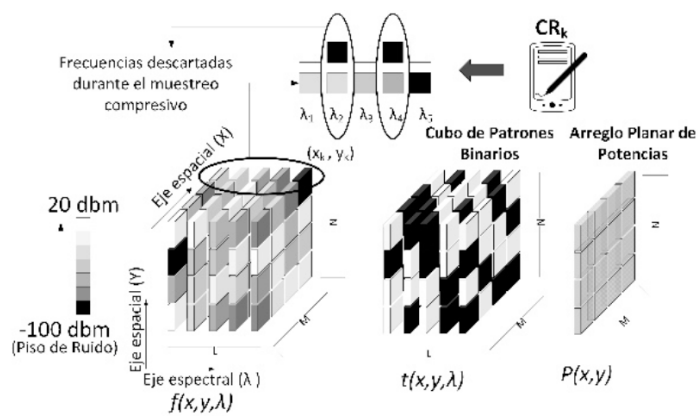

Figura 12. Arquitectura 2: Múltiples patrones binarios. $(j, l)$, mapeada en el PSPA P de dimensión $M \times N, L$ es el número de bandas espectrales disponibles, $T_{j l k}$ es un cubo de datos con los patrones binarios para $0 \leq k \leq L-1, F_{j l k}$ es el cubo de datos completo original con la información de potencia en los planos geográficos y espectral, y $w_{j l}$ es el ruido del sistema.

En este caso el SB genera múltiples patrones binarios, y le solicitará información a los SDRs de la mayoría de posiciones geográficas. Sin embargo, la información solicitada a cada uno difiere en las bandas de frecuencia de las cuales se solicitan niveles de potencia. Una vez el SB tiene la información suministrada por cada SDR procede a conformar el PSPA y reconstruir el cubo de datos.

\section{Arquitectura con desplazamiento del cubo de} datos en la formación del PSPA

La Figura 13 muestra una arquitectura similar a la arquitectura con patrón binario fijo. Sin embargo, en este caso el PSPA se conforma con la combinación lineal de muestras desplazadas en el eje espacial del cubo de datos y los pixeles del patrón binario. Por lo tanto, cada pixel del PSPA es el resultado de la combinación lineal de muestras de potencia que no necesariamente hacen parte del mismo punto geográfico. Note que en este caso la dimensión del PSPA es de $V \times N$ donde $\mathrm{V}=M+L$-1. Esta arquitectura se propone como una analogía exacta con la arquitectura CASSI usada en CSI [21].

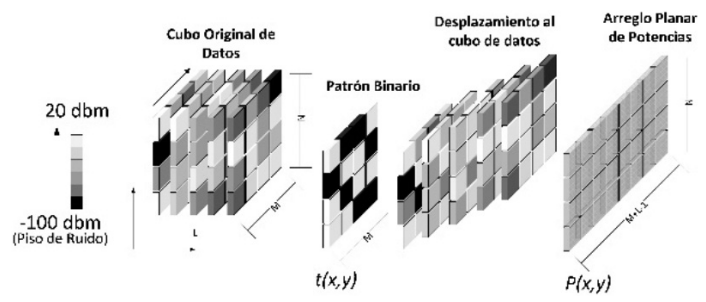

Figura 13. Arquitectura 3: Código de apertura fijo y desplazamiento del cubo de datos.

El PSPA se modela en forma discreta como

$$
P_{j l}=\sum_{k=0}^{L-1} F_{j(l+k)(k)} T_{j(l+k)}+w_{j l},
$$

donde $P_{j l}$ corresponde a los datos de la posición $(j, l)$ del PSPA P de dimensión $V \times N$ donde $V=M+L-1$, $L$ es el número de bandas espectrales disponibles, 
$T_{j l}$ es el patrón binario, $F_{j l k}$ es el cubo de datos completo original con la información de potencia en los planos geográficos y espectral, y $w_{j l}$ es el ruido del sistema, que se puede modelar como una señal de distribución normal.

\section{Reconstrucción del cubo con información de} potencia espacial/espectral del sistema

En CSCR, sea $x$ el cubo multiespectral de intensidades de potencia en RC. Al expresarlo en otra base de representación queda expresado de la forma $f=\Psi_{\mathrm{X}}$ donde $\Psi \in \mathbb{R}^{M \times N}$ corresponde a una matriz de transformación. Por su parte el modelo matemático del PSPA se formula como $\mathrm{p}=\mathrm{Ax}=\mathrm{H} \Psi_{\mathrm{x}}=\mathrm{Hf}$ donde $\mathrm{A}=\mathrm{H} \Psi$ corresponde a la matriz de muestreo. La estructura de la matriz $A$ es tal, que garantiza que $p=A x$ corresponda a un sistema indeterminado de ecuaciones lineales, y depende de los patrones binarios usados para realizar el muestreo organizados matricialmente en $H$, y de la base de representación $\Psi$ de la señal. En [21] se presenta de forma detallada el proceso matemático para la conformación de $A$. Para hacer la reconstrucción del cubo espectral de potencia se debe resolver el problema de optimización

$$
\begin{gathered}
\min _{x, w \in \mathbb{R}^{N}, v \in \mathbb{R}^{M}}\|w\|_{1}+\iota_{E(\in, 0)}(v) \\
\text { sujeto a } w=x \text { y } v=A x-p
\end{gathered}
$$

que corresponde al problema de optimización C-SALSA. En [45] se formula el algoritmo para resolver este problema.

\section{SIMULACIÓN DEL MODELO Y RESULTADOS}

\section{Definición del escenario y restricciones}

Sea un escenario con 30 SDR's ubicados aleatoriamente en un área geográfica con $256 \times 256$ celdas de ubicación que representan un área de 256 metros cuadrados. La primera restricción, por lo tanto, es dada por la cuantificación del área geográfica. Los SDR's pueden transmitir en 8 posibles bandas espectrales. Por lo tanto, el cubo multiespectral original es de $256 \times 256 \times 8$. Cada SDR transmite con una potencia de $20 \mathrm{dBm}$. $\mathrm{La}$ atenuación de potencia sigue el modelo de espacio libre, y el piso de ruido es de $-116 \mathrm{dBm}$.

Se conforma el cubo multiespectral y se hace muestreo compresivo usando el patrón binario mostrado en la Figura 14 a, y posteriormente se construye la matriz que representa el PSPA usando la ecuación (12), (13) o (14), dependiendo de la arquitectura a implementar. Para la construcción del PSPA teniendo en cuenta puntos geográficos diferentes a las ubicaciones de los SDR's, se hacen predicciones de niveles de potencia en toda la malla espacial con base en modelos de propagación.

En la Figura 14 b se muestra el caso de la matriz $\mathrm{P}$ que representa el PSPA calculado usando la ecuación (13). En el caso de la arquitectura 2, se generan 8 patrones binarios distintos con la misma distribución estadística del patrón mostrado en la Figura 14 a para conformar el cubo $t(x, y, \lambda)$ mostrado en la Figura 12.

A partir del PSPA, se construye la matriz $\mathrm{H}$ y se resuelve el problema de optimización mostrado en la ecuación (15) para calcular x. Como base de representación $\Psi$ usa la base Kronecker, que es normalmente usada en problemas CSI [21]. De esta forma se construye la señal dispersa $f=\Psi x$.

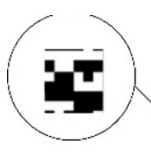

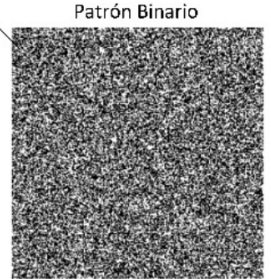

a)

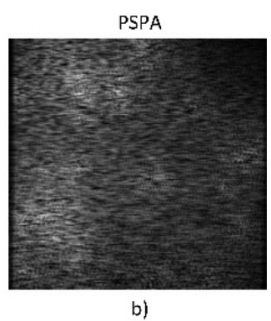

b)
Figura 14.a) Patrón binario con transmitancia de 0,5. b) PSPA calculado.

Finalmente se compara el cubo reconstruido con el original en términos de PSNR entre la imagen espectral $2 \mathrm{D}$ original y reconstruida para cada $\lambda_{k}$. Este proceso se hace para transmitancias del patrón binario desde 0,1 hasta 0,9 , con el propósito de determinar el porcentaje óptimo de muestras a rechazar.

Resultados en la reconstrucción espacio-espectral La Figura 15 muestra el resultado para 4 bandas espectrales, usando la arquitectura 2 , con un patrón binario con transmitancia 0,5. En la Figura 16 se muestra el efecto de la transmitancia del patrón binario en la reconstrucción de los datos. Note que los valores óptimos de transmitancia están alrededor de 0,5. 
Este resultado muestra que para transmitancias mayores de 0,6 la reconstrucción no mejora, es decir, no necesariamente el tomar más muestras permite una mejor reconstrucción de la señal usando el MC multiespectral. Una transmitancia de 0,5 significa que en el MC se descartan como mínimo el 50\\% de muestras de potencia para cada banda espectral. Los resultados también muestran que el desempeño del sistema no es bueno en la arquitectura de patrón binario fijo. Esta arquitectura genera un patrón binario único para todas las bandas espectrales, por lo que se están descartando ciertas posiciones geográficas en el muestreo. Las arquitectura 2 , en cambio, permite la selección aleatoria de diferentes posiciones geográficas en diferentes bandas al contar con múltiples patrones.
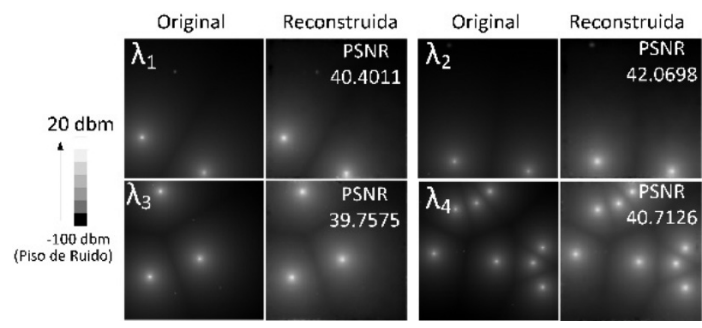

Figura 15. Datos reconstruidos por el gestor espectral.

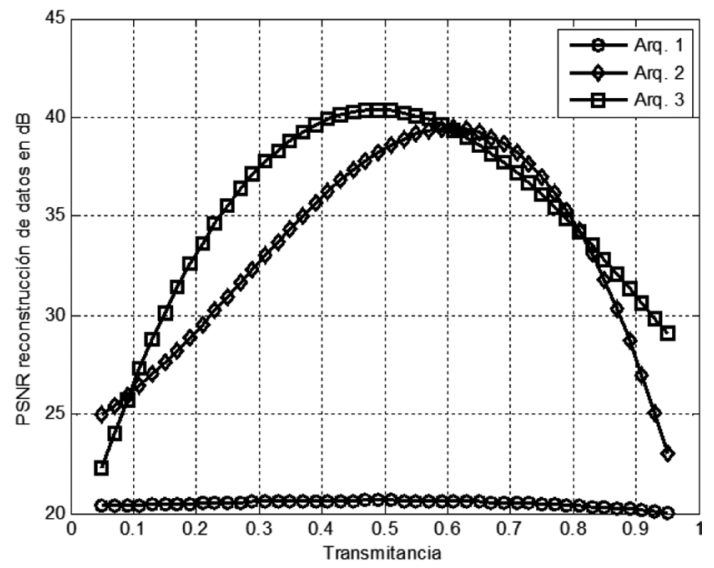

Figura 16. Análisis de transmitancia. Arq.1: Patrón binario fijo. Arq. 2: Múltiples patrones binarios. Arq. 3: Con dispersión de datos.

Resultados en función de la cantidad de datos usados para la reconstrucción

Gracias al modelo descrito con las ecuaciones (12), (13) o (14), todos los datos del cubo espectral quedan mapeados en el PSPA, que para las arquitecturas 1 y 2 es de dimensión $M \times N$, y en el caso de la arquitectura 3 es de dimensión $V \times N$ donde $V=M+L-1$, y $L$ es el número de bandas espectrales disponibles. De acuerdo con estos valores, se calcula una razón de diezmado de datos, la que se define como:

$$
R D=\frac{N D}{N M},
$$

Donde $N D$ corresponde al número total de datos originales y $N M$ corresponde al número de datos usados para la reconstrucción. La Tabla 1 muestra los resultados obtenidos, para 8 bandas espectrales, usando la arquitectura 2 del modelo multiespectral propuesto, en comparación con las razones de muestreo usadas en trabajos previos que usan interpolación espacial [28], y MC usando el algoritmo “Orthogonal Matching Pursuit" (OMP) [31].

Tabla 1. Razón de diezmado y error promedio en la reconstrucción en tres modelos de reconstrucción de mapas de cartografía de interferencia - 8 bandas espectrales.

\begin{tabular}{|l|r|r|l|l|}
\cline { 2 - 5 } \multicolumn{1}{c|}{} & \multicolumn{1}{c|}{ ND } & NM & RD & Error \\
\hline Modelo MC-Multiespectral & 524.288 & 32.768 & 16 & $24 \mathrm{dBm}$ \\
\hline Modelo con MC - OMP & 5.000 & 400 & 12,5 & $2 \mathrm{dBm}^{*}$ \\
\hline $\begin{array}{l}\text { Modelo con interpolación } \\
\text { espacial }\end{array}$ & 80.000 & 20.000 & 4 & $7 \mathrm{dBm}^{+}$ \\
\hline
\end{tabular}

* Para 50 sensores (50 datos por banda).

+ Para $50 \%$ de muestras por banda espectral.

Se puede observar que con el modelo propuesto se mejora la razón de diezmado, lo que significa que se necesitan menos datos para transmitir o almacenar la información. Una razón de diezmado de 16, equivale a $6,25 \%$ del número de muestras totales. Sin embargo, el error de reconstrucción es mayor, debido a que los datos de todas las bandas se mapean en un solo plano, y el proceso de reconstrucción abarca datos tanto espaciales como espectrales, mientras que en los otros trabajos solo se procesa una banda a la vez y la reconstrucción es solamente en el dominio espacial.

\section{CONCLUSIONES}

Las técnicas de MC y de CSI usadas en el tratamiento de imágenes multiespectrales son viables en el 
sensado espectral en RC, con el objetivo de disminuir la cantidad de muestras de potencia muestreadas para poder identificar las porciones espectrales que no están siendo utilizadas o que generan poca interferencia. Se propone un modelo de sensado multiespectral para radio cognitiva (CSCR) donde un gestor espectral $(\mathrm{SB})$ recibe de forma cooperativa muestras compresivas de potencia a diferentes bandas espectrales de diferentes SDR y usando muestreo comprimido (MC) caracteriza la ocupación espectral en un área de cobertura determinada. El sistema entrega un cubo de datos de $M \times N \times L$ donde $L$ es el número de bandas espectrales disponibles y de cada una se tiene un arreglo espacial de $M \times N$ con información de intensidad de potencia en el área geográfica. Esta información le permitirá al SB decidir qué frecuencia asignar a un SDR que le solicite servicio, ubicado en un punto geográfico conocido, sin que cause interferencia con otros usuarios. El MC se realiza usando patrones binarios de transmitancia variable que definen qué muestras capturar y qué muestras descartar. Se presentaron tres posibles arquitecturas para implementar CSCR. Los resultados de las simulaciones muestran que es posible reconstruir la información con un alto grado de precisión, usando patrones binarios de transmitancia entre 0,4 y 0,6 , lo que significa un ahorro entre $40 \%$ y $60 \%$ de las muestras adquiridas por cada banda, y el posterior mapeo de la información en el PSPA, lo que permite la reconstrucción de la información espectral y espacial con solo el 6,25\% de la cantidad original de datos. Esto permitirá optimizar los tiempos de operación del SB en el sensado espectral.

\section{REFERENCIAS}

[1] M.M. Buddhikot. "Understanding dynamic spectrum access: Models, taxonomy and challenges". G. Salini Calderón y P. Pérez Jara. "Estudio de series temporales de contaminación ambiental mediante técnicas de redes neuronales artificiales". New Frontiers in Dynamic Spectrum Access Networks. pp. 649-663. DySPAN, 2nd IEEE International Symposium. 2007.

[2] Li-Chun Wang and S. Rangapillai. "A survey on green $5 \mathrm{~g}$ cellular networks". Signal Processing and Communications (SPCOM), 2012 International Conference on, pp. 1-5, 2012.
I.F. Akyildiz, Won-Yeol Lee, Mehmet C. Vuran, and S. Mohanty. "A survey on spectrum management in cognitive radio networks". Communications Magazine, IEEE. Vol. 46 $\mathrm{N}^{\mathrm{o}}$ 4, pp. 40-48. 2008.

[4] J.M. Alfonso and L.B. Agudelo. "Centralized spectrum broker and spectrum sensing with compressive sensing techniques for resource allocation in cognitive radio networks". IEEE Latin-America Conference on Communications, pp. 1-6. November, 2013.

[5] E.J. Candes and M.B. Wakin. "An introduction to compressive sampling". Signal Processing Magazine, IEEE. Vol. 25 Issue 2, pp. 21-30. March, 2008.

[6] Yonina C. Eldar and Gitta Kutyniok. "Compressed Sensing: Theory and Applications". Cambridge. 2012.

[7] Dongeun Lee and Jaesik Choi. "Learning dynamic compressive sensing models", pp. 1-16. 2015.

[8] Ricard G. Baraniuk. "Compressive sensing". IEEE Signal Processing Magazine, pp. 118124. 2007.

[9] David L. Donoho. "Compressed sensing" IEEE Transactions on Information Theory. Vol. 52 Issue 4, pp. 1289-1306. 2006.

[10] Jarvis Haupt, Rui Castro, Robert Nowak, Gerald Fudge y Alex Yeh. "Compressive Sampling for Signal Classification. In 2006 Fortieth Asilomar Conference on Signals, Systems and Computers", pp. 1430-1434. IEEE. 2006.

[11] Saad Qaisar. "Compressive sensing: From theory to applications, a survey". Journal of communications and networks. Vol. 15, pp. 443-455. 2013.

[12] Wotao Yin Zhu Han, Husheng Li. "Compressive Sensing for Wireless Networks". Vol. 1. Cambridge University Press, New York. 2013.

[13] Masaaki Nagahara Kasunori Hayashi and Toshiyiky Tanaka. "A user's guide to compressed sensing for communications systems", IEICE Trans. Commun. E96-B(3), pp. 685-712. Marzo 2013.

[14] C.R. Berger, Zhaohui Wang, Jianzhong Huang, y Shengli Zhou. "Application of compressive sensing to sparse channel estimation". Communications Magazine. IEEE. Vol. 48, Issue 11, pp. 164-174. 2010.

[15] A. Sayeed, R. Nowaka and W.U. Bajwa. "Compressed sensing of wireless channels 
in time, frequency, and space". Signals, Systems and Computers. 2008.

[16] Changyong Pan and Linglong Dai. "Time domain synchronous ofdm based on compressive sensing: A new perspective". Global Communications Conference (GLOBECOM). 2012.

[17] A. Akbarpour-Kasgari and S. N. Esfahani. "Compressed receiver for ultra-wideband communication". Electrical Engineering (ICEE). 2013.

[18] C.S. Lu y S.Y. Kuo C.M. Yu. "Compressed sensing-based clone identification in sensor networks". IEEE Transactions on Wireless Communications. Vol. 99, pp. 1-14. 2016.

[19] M.B. Hawes and W. Liu. "Robust sparse antenna array design via compressive sensing". Digital Signal Processing (DSP). 2013.

[20] M. Grangetto and M. Sereno R. Gaeta. "Local access to sparse and large global information in P2P networks: a case for compressive sensing". Peer-to-Peer Computing (P2P). 2010.

[21] Z. Tian and G.B. Giannakis. "Compressed sensing for wideband cognitive rádios". IEEE International Conference on Acoustics, Speech and Signal Processing - ICASSP ' 07. Vol. 4, pp. IV-1357-IV-1360. Abril 2007.

[22] F. Ayhan Sakarya, G.S. Nagel, L.H. Tran, and J.A. Molnar. Wideband. "Compressed Sensing for Cognitive Radios". MILCOM 2011, Military Communications Conference, pp. 31-36. Nov. 2011.

[23] L. Zhou and H. Man. "Cooperative compressive spectrum sensing in cognitive radio based on w-omp". In MILCOM 2013 - 2013 IEEE Military Communications Conference, pp. 11871192, Nov. 2013.

[24] G.R. Arce, D.J. Brady, L. Carin, H. Arguello and D.S. Kittle. "Compressive coded aperture spectral imaging: An introduction”, Signal Processing Magazine, IEEE. Vol. 31 Issue 1, pp. 105-115. Enero 2014.

[25] H. Arguello y G.R. Arce. "Colored coded aperture design by concentration of measure in compressive spectral imaging. Image Processing”. IEEE Transactions on, Vol. 23 Issue 4, pp. 1896-1908. Abril 2014.

[26] Xing Lin, Yebin Liu, Jiamin Wu, y Qionghai Dai. "Spatial-spectral encoded compressive hyperspectral imaging". ACMTrans. Graph. Vol.33 Issue 6, pp. 233: 1-233:11, November 2014.
[27] Claudia V. Correa, Henry Arguello and Gonzalo R. Arce. "Snapshot colored compressive spectral imager". J. Opt. Soc. Am. A. Vol. 32 Issue 10, pp. 1754-1763. Oct 2015.

[28] Afef B. Sana B., Berna S. Paul H. "Informed spectrum usage in cognitive radio networks: Interference cartography" IEEE 19th International Symposium on Personal, Indoor and Mobile Radio Communications, Cannes, pp. 1-5, 2008.

[29] R. Janne, N. Jad and M. Petri. "Demonstrating radio environment map construction from massive data sets". IEEE International Symposium on Dynamic Spectrum Access Networks, Bellevue, WA, pp. 266-267, 2012.

[30] B. Juan, M. Gonzalo and B. Georgios. "GroupLasso on Splines for Spectrum Cartography". IEEE Transactions on Signal Processing. Vol. 59 Issue 10, pp. 4648-4663. Oct. 2011.

[31] B.A. Jayawickrama, E. Dutkiewicz, I. Oppermann, G. Fang and J. Ding, "Improved performance of spectrum cartography based on compressive sensing in cognitive radio networks". IEEE International Conference on Communications (ICC), Budapest, pp. 5657-5661. 2013.

[32] Marco H. "Spectrum Occupancy Measurements: A Survey and Use of Interference Maps". In IEEE Communications Surveys \& Tutorials. Vol. $18 \mathrm{~N}^{\circ}$ 4, pp. 2386-2414. Fourthquarter 2016.

[33] H Nyquist. "Certain topics in telegraph transmission theory". American Institute of Electrical Engineers, Transactions of the. Vol. 47, pp. 617-644. 1928.

[34] Joel A. Tropp. "Greed is good: Algorithmic results for sparce approximation". IEEE Transactions on Information Theory. Vol. 50 $\mathrm{N}^{\circ} 10.2004$.

[35] Scott Shaobing Chen, David L. Donoho, y Michael a. Saunders. "Atomic Decomposition by Basis Pursuit", SIAM Journal on Scientific Computing. Vol. $20 \mathrm{~N}^{\mathrm{o}}$ 1, pp. 33-61. 1998.

[36] Yi Chen, Nasser M Nasrabadi, y Trac D Tran. "Hyperspectral Image Classification Using DictionaryBased Sparse Representation", IEEE transactions on geoscience and remote sensing. Vol. $49 \mathrm{~N}^{\mathrm{o}}$ 10-2, pp. 3973-3985. 2011.

[37] Karlheinz Gröchenig. "Foundations of timefrequency analysis", Springer Science \& Business Media, 2013. 
[38] Felix Krahmer, Deanna Needell, and Rachel Ward. "Compressive sensing with redundant dictionaries and structured measurements", pp. 1-21, 2015.

[39] E. Candes y J. Romberg. "L1-magic: Recovery of sparse signals via convex programming" Tecnical Report, Caltech., Octubre 2005.

[40] J. Bobin y E. Candes S. Becker. "Nesta: A fast and accurate first-order method for sparse recovery", SIAM J. Imaging Sci. Vol. 4, Issue 1, pp. 1-39, 2011.

[41] E.V.D. Berg and M.P. "Friedlander. Probing the pareto frontier for basis pursuit solutions", SIAM J. Imaging Sci. Vol. 2, Issue 1, pp. 143168, Marzo 2008.

[42] A. Beck y M. Teboulle. "A fast iterative shrinkage-thresholding algorithm for lineal inverse problems", SIAM J. Imaging Sci. Vol. 2, Issue 1, pp. 183-202. 2009.

[43] M.A.T. Figueiredo, R.D. Nowak, y S.J. Wright. "Gradient projection for sparse reconstruction: Application to compressed sensing and other inverse problems", Selected Topics in Signal Processing, IEEE Journal of, Vol. 1, Issue 4, pp. 586-597. Dic 2007.

[44] J.M. Bioucas-Dias y M.A.T. Figueiredo. "A new twist: Two-step iterative shrinkage/ thresholding algorithms for image restoration", IEEE Transactions on Image Processing. Vol. 16 No 12, pp. 2992-3004. Dic. 2007.

[45] M.V. Afonso, J.M. Bioucas-Dias, y M.A. T. Figueiredo. "An augmented lagrangian approach to the constrained optimization formulation of imaging inverse problems" IEEE Transactions on Image Processing, Vol. 20, Issue 3, pp. 681-695. Marzo 2011.

[46] Mohammad Golbabaee, Simon Arberet, y Pierre Vandergheynst. "Compressive Source Separation: Theory and Methods for Hyperspectral Imaging" IEEE Transactions on Image Processing, Vol. 22, Issue 12, pp. 5096-5110.Dic. 2013.

[47] A Zare, J Bolton, J Chanussot, y P Gader. "Foreword to the Special Issue on Hyperspectral Image and Signal Processing", Selected Topics in Applied Earth Observations and Remote Sensing, IEEE Journal of, Vol. 7 , Issue 6, pp. 1841-1843. 2014.
[48] Elhadi Adam, Onisimo Mutanga, y Denis Rugege. "Multispectral and hyperspectral remote sensing for identification and mapping of wetland vegetation: A review" Wetlands Ecology and Management, Vol. 18, Issue 3, pp. 281-296. 2010.

[49] J. Nocedal and S. J. Wright. "Numerical Optimization", New York:Springer-Verlag, 2nd edition, 2006.

[50] J. Eckstein y D. Bertsekas. "On the douglas-rachford splitting method and the proximal point algorithm for maximal monotone operators", Math. Program. Vol. 5, pp. 293-318. 1992.

[51] O. Ileri, D. Samardzija, y N.B. Mandayam. "Demand responsive pricing and competitive spectrum allocation via a spectrum server" New Frontiers in Dynamic Spectrum Access Networks, DySPAN, First IEEE International Symposium, pp. 194-202, 2005.

[52] A.M. Wyglinski, M. Nekovee, y Y.T. Hou. "Cognitive Radio Communications and Networks: Principles and Practice" Academic Press, 2009.

[53] M. Kaplan y F. Buzluca. "A dynamic spectrum decision scheme for heterogeneous cognitive radio networks", Computer and Information Sciences, ISCIS, 24th International Symposium on, pp. 697-702, 2009.

[54] A. Chowdhury, Hung-Chang Chien, Yu-Ting Hsueh, y Gee-Kung Chang. "Advanced system technologies and field demonstration for in-building optical-wireless network with integrated broadband services", Lightwave Technology, Journal of, Vol. 27, Issue 12, pp. 1920-1927. Junio 15, 2009.

[55] M.Z. Chowdhury, Sunwoong Choi, Yeong Min Jang, Kap-Suk Park, y Geun Il Yoo. "Dynamic sla negotiation using bandwidth broker for femtocell networks", Ubiquitous and Future Networks, ICUFN, First International Conference on, pp. 12-15, 2009.

[56] J.W. Mwangoka, P. Marques, y J. Rodríguez. "Broker based secondary spectrum trading", Cognitive Radio Oriented Wireless Networks and Communications (CROWNCOM), Sixth International ICST Conference on, pp. 186-190, 2011. 